\title{
Acute appendicitis as a complication of treatment of a patient with Rheumatoid Arthritis?
}

\author{
Authors: Damir Mišura ${ }^{1}$, Luka Matanović ${ }^{1}$, Porin Perić ${ }^{1,2}$ \\ ${ }^{1}$ School of Medicine, University of Zagreb, Croatia \\ ${ }^{2}$ Hospital Centre Zagreb, Department of Rheumatology and Rehabilitation, Zagreb, Croatia \\ DOI: https://doi.org/10.26800/LV-142-supp5-26
}

\section{Background:}

Rheumatoid Arthritis (RA) is a chronic, systemic immune disease which can affect many organs, but it mainly affects the synovial joints. It can occur at any age, but is most frequent in the third and fourth decade of life.

\section{Case presentation:}

We are presenting a case of a thirty-two year old patient with RA who developed acute abdomen during treatment with a biological medication. In November 2009, treatment with adalimumab was started. At the time, patient was also treated with methotrexate (10 mg per week), methylprednisolone and diclofenac $(8 / 100 \mathrm{mg} /$ day). After six weeks of treatment with adalimumab, the patient had to be urgently surgically treated due to acute appendicitis. In the early postsurgical period, acute abdomen develops again. Sigmoidectomy was performed, as a treatment for the "sigmoid colon block" and subsequently, sigmoidostomy was done. Four weeks later mechanical ileus occurred, due to adhesions, and adhesiolysis was performed. Adalimumab was discontinued after first surgical treatment. Although gastrointestinal disorders are common in patients with RA, it is very rarely that a surgical procedure is needed. On the other hand, there is much proof that suggests unfavorable effect of non- steroid antirheumatic and glucocorticoid drugs on the digestive system. Acute appendicitis has been described as a serious infection during adalimumab therapy in patients with ankylosing spondylitis. There is many data that suggest the risk of developing infections due to treatment with drugs that neutralize TNF-alpha bioactivity. Most of the cases develop in the first six months of the drug use.

\section{Conclusion:}

Our patient developed a serious infection in the gastrointestinal system that required hospitalization and emergency surgery. This is probably due to the treatment with a biological medicine adalimumab, although it is difficult to roll out completely the effect of the severity of the disease itself and also the simultaneous use of NSAID and glucocorticoids.

Keywords: acute appendicitis, adalimumab, rheumatoid arthritis 